TRANSACTIONS OF THE

AMERICAN MATHEMATICAL SOCIETY

Volume 361, Number 6, June 2009, Pages 3359-3371

S 0002-9947(09)04677-7

Article electronically published on January 28, 2009

\title{
NON-DEGENERACY OF WIENER FUNCTIONALS ARISING FROM ROUGH DIFFERENTIAL EQUATIONS
}

\author{
THOMAS CASS, PETER FRIZ, AND NICOLAS VICTOIR
}

\begin{abstract}
Malliavin Calculus is about Sobolev-type regularity of functionals on Wiener space, the main example being the Itô map obtained by solving stochastic differential equations. Rough path analysis is about strong regularity of the solution to (possibly stochastic) differential equations. We combine arguments of both theories and discuss the existence of a density for solutions to stochastic differential equations driven by a general class of non-degenerate Gaussian processes, including processes with sample path regularity worse than Brownian motion.
\end{abstract}

\section{INTRODUCTION}

It is a basic question in probability theory whether a given stochastic process $\left\{Y_{t}: t \geq 0\right\}$ with values in some Euclidean space admits, at fixed positive times, a density with respect to Lebesgue measure. In a non-degenerate Markovian setting - ellipticity of the generator - an affirmative answer can be given using Weyl's lemma as discussed in McKean's classical 1969 text [23. Around the same time, Hörmander's seminal work on hypoelliptic partial differential operators enabled probabilists to obtain criteria for existence (and smoothness) for densities of certain degenerate diffusions. This dependence on the theory of partial differential equations was removed when P. Malliavin devised a purely probabilistic approach, perfectly adapted to prove existence and smoothness of densities.

We recall some key ingredients of Malliavin's machinery, known as Malliavin Calculus or stochastic calculus of variations. Most of it can be formulated in the setting of an abstract Wiener space $(W, \mathcal{H}, \mu)$. The concept is standard [3, 22, 24, 28, as is the notion of a weakly non-degenerate $\mathbb{R}^{e}$-valued Wiener functional $\varphi$ which has the desirable property that the image measure $\varphi_{*} \mu$ is absolutely continuous with respect to Lebesgue measure on $\mathbb{R}^{e}$. (Functionals which are non-degenerate have a smooth density.) Precise definitions are given later in the text.

Given these abstract tools, we turn to the standard Wiener space $C_{0}\left([0,1], \mathbb{R}^{d}\right)$ equipped with Wiener measure. From Itô's theory, we can realize diffusion processes by solving the stochastic differential equation

$$
d Y=\sum_{i=1}^{d} V_{i}(Y) \circ d B^{i}+V_{0}(Y) d t \equiv V(Y) \circ d B+V_{0}(Y) d t, \quad Y(0)=y_{0} \in \mathbb{R}^{e},
$$

Received by the editors May 11, 2007 and, in revised form, November 7, 2007.

2000 Mathematics Subject Classification. Primary 60G15, 60H07, 60H10, 60K99.

Key words and phrases. Malliavin Calculus, rough paths analysis. 
driven by a $d$-dimensional Brownian motion $B$ along sufficiently well-behaved (drift - resp. diffusion) vector fields $V_{0}, \ldots, V_{d}$. The Itô-map $B \mapsto Y$ is notorious for its lack of strong regularity properties, which rules out the use of any Fréchet-calculus. On the positive side, it is smooth in a Sobolev sense on Wiener space ("smooth in the sense of Malliavin"). Under condition

$$
(E): \operatorname{span}\left[V_{1}, \ldots, V_{d}\right]_{y_{0}}=\mathcal{T}_{y_{0}} \mathbb{R}^{e} \cong \mathbb{R}^{e}
$$

or a less restrictive Hörmander's condition (allowing all Lie brackets of $V_{0}, \ldots, V_{d}$ in spanning $\left.\mathcal{T}_{y_{0}} \mathbb{R}^{e}\right)$ the solution map $B \mapsto Y_{t}$, for $t>0$, is non-degenerate, and one reaches the desired conclusion that $Y_{t}$ has a (smooth) density. This line of reasoning due to $\mathrm{P}$. Malliavin provides a direct probabilistic approach to the study of transition densities and has found applications from stochastic fluid dynamics to interest rate theory. It also shows that the Markovian structure is not essential, and one can, for instance, adapt these ideas to study densities of Itô-diffusions as was done by Kusuoka and Stroock in [17.

Our interest lies in stochastic differential equations of type

$$
d Y=V(Y) d X+V_{0}(Y) d t,
$$

where $X$ is a multi-dimensional Gaussian process. Such differential equations arise, for instance, in financial mathematics [2, 10, 11] or as model for studying ergodic properties of non-Markovian systems [12. Assuming momentarily enough sample path regularity so that (1.1) makes sense path-by-path by Riemann-Stieltjes (or Young) integration, the question of whether or not $Y_{t}$ admits a density is important and far from obvious. To the best of our knowledge, all results in that direction are restricted to fractional Brownian motion with Hurst parameter $H>1 / 2$. Existence of a density for $Y_{t}, t>0$, was established in 25] under condition (E). Smoothness results then appeared in [26, and under Hörmander's condition in [1.

The purpose of this paper is to give a first demonstration of the powerful interplay between Malliavin Calculus and rough path analysis. After some remarks on $\mathcal{H}$ differentiability and a representation of the Malliavin covariance in terms of a $2 \mathrm{D}$ Young integral, we show that weak non-degeneracy (in the sense of Malliavin) of solutions to (1.1) at times $T>0$, and hence existence of a density, remains valid in an almost generic sense. Our assumptions are

- The vector fields $V=\left(V_{1}, \ldots, V_{d}\right)$ at $y_{0}$ span $\mathcal{T}_{y_{0}} \mathbb{R}$, i.e. condition (E).

- The continuous, centered Gaussian driving signal $X$ is such that the stochastic differential equation (1.1) makes sense as a rough differential equation (RDE), [18, 21]. Applied in our context, this represents a unified framework which covers at once Gaussian signals with nice sample paths (such as fBM with $H>1 / 2$ ), Brownian motion, Gaussian (semi-)martingales, and last but not least Gaussian signals with sample path regularity worse than Brownian motion, provided there exists a sufficiently regular stochastic area 1

- The Gaussian driving signal is sufficiently non-degenerate, which is clearly needed to rule our examples such as $X \equiv 0$ or the Brownian bridge $X_{t}=$ $B_{t}(\omega)-(t / T) B_{T}(\omega)$.

Smoothness of densities remains an open problem; some technical remarks about the difficulties involved are found in the last section.

\footnotetext{
${ }^{1}$ For orientation, fractional Brownian motion is covered for any $H>1 / 3$.
} 


\section{Preliminaries on ODE and RDEs}

2.1. Controlled ordinary differential equations. Consider the ordinary differential equations, driven by a smooth $\mathbb{R}^{d}$-valued signal $f=f(t)$ along sufficiently smooth and bounded vector fields $V=\left(V_{1}, \ldots, V_{d}\right)$ and a drift vector field $V_{0}$

$$
d y=V(y) d f+V_{0}(y) d t, y\left(t_{0}\right)=y_{0} \in \mathbb{R}^{e} .
$$

We call $U_{t \leftarrow t_{0}}^{f}\left(y_{0}\right) \equiv y_{t}$ the associated flow. Let $J$ denote the Jacobian of $U$. It satisfies the ODE obtain by formal differentiation w.r.t. $y_{0}$. More specifically,

$$
a \mapsto\left\{\frac{d}{d \varepsilon} U_{t \leftarrow t_{0}}^{f}\left(y_{0}+\varepsilon a\right)\right\}_{\varepsilon=0}
$$

is a linear map from $\mathbb{R}^{e} \rightarrow \mathbb{R}^{e}$, and we let $J_{t \leftarrow t_{0}}^{f}\left(y_{0}\right)$ denote the corresponding $e \times e$ matrix. It is immediate to see that

$$
\frac{d}{d t} J_{t \leftarrow t_{0}}^{f}\left(y_{0}\right)=\left[\frac{d}{d t} M^{f}\left(U_{t \leftarrow t_{0}}^{f}\left(y_{0}\right), t\right)\right] \cdot J_{t \leftarrow t_{0}}^{f}\left(y_{0}\right),
$$

where $\cdot$ denotes matrix multiplication and

$$
\frac{d}{d t} M^{f}(y, t)=\sum_{i=1}^{d} V_{i}^{\prime}(y) \frac{d}{d t} f_{t}^{i}+V_{0}^{\prime}(y) .
$$

Note that $J_{t_{2} \leftarrow t_{0}}^{f}=J_{t_{2} \leftarrow t_{1}}^{f} \cdot J_{t_{1} \leftarrow t_{0}}^{f}$. We can consider Gateaux derivatives in the driving signal and define

$$
D_{h} U_{t \leftarrow 0}^{f}=\left\{\frac{d}{d \varepsilon} U_{t \leftarrow 0}^{f+\varepsilon h}\right\}_{\varepsilon=0} .
$$

One sees that $D_{h} U_{t \leftarrow 0}^{f}$ satisfies a linear ODE, and the variation of constants formula leads to

$$
D_{h} U_{t \leftarrow 0}^{f}\left(y_{0}\right)=\int_{0}^{t} \sum_{i=1}^{d} J_{t \leftarrow s}^{f}\left(V_{i}\left(U_{s \leftarrow 0}^{f}\right)\right) d h_{s}^{i} .
$$

2.2. Rough differential equations. Let $p \in(2,3)$. A geometric $p$-rough path $\mathbf{x}$ over $\mathbb{R}^{d}$ is a continuous path on $[0, T]$ with values in $G^{2}\left(\mathbb{R}^{d}\right)$, the step- 2 nilpotent group over $\mathbb{R}^{d}$, of finite $p$-variation relative to $d$, the Carnot-Caratheodory metric on $G^{2}\left(\mathbb{R}^{d}\right)$, i.e.

$$
\sup _{n} \sup _{t_{1}<\ldots<t_{n}} \sum_{i} d\left(\mathbf{x}_{t_{i}}, \mathbf{x}_{t_{i+1}}\right)^{p}<\infty .
$$

Following [18, 6, 7, 9] we realize $G^{2}\left(\mathbb{R}^{d}\right)$ as the set of all $(a, b) \in \mathbb{R}^{d} \oplus \mathbb{R}^{d \times d}$ for which $\operatorname{Sym}(b) \equiv a^{\otimes 2} / 2$. (This point of view is natural: a smooth $\mathbb{R}^{d}$-valued path $x=\left(x_{t}^{i}\right)_{i=1, \ldots, d}$, enhanced with its iterated integrals $\int_{0}^{t} \int_{0}^{s} d x_{u}^{i} d x_{s}^{j}$, gives canonically rise to a $G^{2}\left(\mathbb{R}^{d}\right)$-valued path.) Given $(a, b) \in G^{2}\left(\mathbb{R}^{d}\right)$ one gets rid of the redundant $\operatorname{Sym}(b)$ by $(a, b) \mapsto\left(a, b-a^{\otimes} / 2\right) \in \mathbb{R}^{d} \oplus s o(d)$. Applied to $x$ enhanced with its iterated integrals over $[0, t]$, this amounts to looking at the path $x$ and its (signed) areas $\int_{0}^{t}\left(x_{s}^{i}-x_{0}^{i}\right) d x_{s}^{j}-\int_{0}^{t}\left(x_{s}^{j}-x_{0}^{j}\right) d x_{s}^{i}, i, j \in\{1, \ldots, d\}$. Without going into too much detail, the group structure on $G^{2}\left(\mathbb{R}^{d}\right)$ can be identified with the (truncated) tensor multiplication and is relevant as it allows one to relate algebraically the path and area increments over adjacent intervals; the mapping $(a, b) \mapsto\left(a, b-a^{\otimes} / 2\right)$ maps the Lie group $G^{2}\left(\mathbb{R}^{d}\right)$ to its Lie algebra; at last, the Carnot-Caratheodory 
metric is defined intrinsically as a (left-)invariant metric on $G^{2}\left(\mathbb{R}^{d}\right)$ and satisfies $|a|+|b|^{1 / 2} \lesssim d((0,0),(a, b)) \lesssim|a|+|b|^{1 / 2}$.

One can then think of a geometric $p$-rough path $\mathbf{x}$ as a path $x:[0, T] \rightarrow \mathbb{R}^{d}$ enhanced with its iterated integrals (equivalently: area integrals), although the latter need not make classical sense. For instance, almost every joint realization of Brownian motion and Lévy's area process is a geometric $p$-rough path [21, 6, [5]. The Lyons theory of rough paths [20, 21, 9] then gives deterministic meaning to the rough differential equation (RDE)

$$
d y=V(y) d \mathbf{x}
$$

for $\operatorname{Lip}^{\gamma}$-vector fields (in the sense of Stein), $\gamma>p$. By considering the space-time rough path $\tilde{\mathbf{x}}=(t, \mathbf{x})$ and $\tilde{V}=\left(V_{0}, V_{1}, \ldots, V_{d}\right)$, this form is general enough to cover differential equations with drift 2 The solution induces a flow $y_{0} \mapsto U_{t \leftarrow t_{0}}^{\mathbf{x}}\left(y_{0}\right)$. The Jacobian $J_{t \leftarrow t_{0}}^{\mathbf{x}}$ of the flow exists and satisfies a linear RDE, as does the directional derivative

$$
D_{h} U_{t \leftarrow 0}^{\mathbf{X}}=\left\{\frac{d}{d \varepsilon} U_{t \leftarrow 0}^{T_{\varepsilon h} \mathbf{x}}\right\}_{\varepsilon=0}
$$

for a smooth path $h$. If $\mathbf{x}$ arises from a smooth path $x$ together with its iterated integrals, the translated rough path $T_{h} \mathbf{x}$ is nothing but $x+h$ together with its iterated integrals. In the general case, we assume $h \in C^{q \text {-var }}$ with $1 / p+1 / q>$ 1 , the translation $T_{h} \mathbf{x}$ can be written in terms of $\mathbf{x}$ and cross-integrals between $\pi_{1}\left(\mathbf{x}_{0, .}\right)=: x$ and the perturbation $h$. (These integrals are well-defined Youngintegrals.)

Proposition 1. Let $\mathbf{X}$ be a geometric p-rough path over $\mathbb{R}^{d}$ and $h \in C^{q \text {-var }}$ $\left([0,1], \mathbb{R}^{d}\right)$ such that $1 / p+1 / q>1$. Then

$$
D_{h} U_{t \leftarrow 0}^{\mathbf{X}}\left(y_{0}\right)=\int_{0}^{t} \sum_{i=1}^{d} J_{t \leftarrow s}^{\mathbf{X}}\left(V_{i}\left(U_{s \leftarrow 0}^{\mathbf{X}}\right)\right) d h_{s}^{i},
$$

where the right hand side is well-defined as Young integral.

Proof. At least when $\gamma>p+1$, both $J_{t \leftarrow 0}^{\mathbf{X}}$ and $D_{h} U_{t \leftarrow 0}^{\mathbf{X}}$ satisfy (jointly with $U_{t \leftarrow 0}^{\mathbf{X}}$ ) an RDE driven by $\mathbf{X}$. This is an application of Lyons' limit theorem and discussed in detail in [19, 20]. A little care is needed, since the resulting vector fields now have linear growth. It suffices to rule out explosion so that the problem can be localized. The needed remark is that $J_{t \leftarrow 0}^{\mathbf{X}}$ also satisfy a linear RDE of the form

$$
d J_{t \leftarrow 0}^{\mathbf{X}}=d \mathbf{M}^{\mathbf{X}} \cdot J_{t \leftarrow 0}^{\mathbf{X}}\left(y_{0}\right),
$$

where $d \mathbf{M}^{\mathbf{X}}=V^{\prime}\left(U_{t \leftarrow 0}^{\mathbf{X}}\left(y_{0}\right)\right) d \mathbf{X}$. Explosion can be ruled out by direct iterative expansion and estimates of the Einstein sum as in [18].

\section{RDES DRIVEn By GaUssian Signals}

We consider a continuous, centered Gaussian process with independent components $X=\left(X^{1}, \ldots, X^{d}\right)$ started at zero. This gives rise to an abstract Wiener space $(W, \mathcal{H}, \mu)$, where $W=\overline{\mathcal{H}} \subset C_{0}\left([0, T], \mathbb{R}^{d}\right)$. Note that $\mathcal{H}=\bigoplus_{i=1}^{d} \mathcal{H}^{(i)}$ and recall that elements of $\mathcal{H}$ are of the form $h_{t}=\mathbb{E}\left(X_{t} \xi(h)\right)$, where $\xi(h)$ is a Gaussian

${ }^{2}(1)$ If $\mathbf{x}$ lifts an $\mathbb{R}^{d}$-valued path $x$, then $\tilde{\mathbf{x}}$ is construct with cross-integrals of type $\int x d t, \int t d x$, all of which are canonically defined. (2) Including $V_{0}$ in the collection $\tilde{V}$ leads to suboptimal regularity requirements for $V_{0}$ which could be avoided by a direct analysis. 
random variable. The ("reproducing kernel") Hilbert-structure on $\mathcal{H}$ is given by $\left\langle h, h^{\prime}\right\rangle_{\mathcal{H}}:=\mathbb{E}\left(\xi(h) \xi\left(h^{\prime}\right)\right)$.

Existence of a Gaussian geometric $p$-rough path above $X$ is tantamount to the existence of certain Lévy area integrals. From the point of view of Stieltjes integration, the existence of Lévy's area for Brownian motion is a miracle. In fact, a subtle cancellation due to orthogonality of increments of Brownian motion is responsible for convergence, and this suggests that processes with sufficiently fast decorrelation of their increments will also give rise to a stochastic Lévy area. The resulting technical conditions appears in 21 for instance. For Gaussian processes, a cleaner (and slightly weaker) condition can be given in terms of the $2 D$ variation properties of the covariance function $R(s, t)=\mathbb{E}\left(X_{s} \otimes X_{t}\right)=\operatorname{diag}\left(R^{(1)}, \ldots, R^{(k)}\right)$. The assumption, writing $X_{t, t^{\prime}}:=X_{t^{\prime}}-X_{t}$,

$$
|R|_{\rho \text {-var; }[0, T]^{2}}^{\rho}:=\sup _{D=\left(t_{i}\right)} \sum_{i, j}\left|\mathbb{E}\left(X_{t_{i}, t_{i+1}} X_{t_{j}, t_{j+1}}\right)\right|^{\rho}<\infty
$$

for $\rho<2$ is known 8 to be sufficient (and essentially necessary) for the existence of a natural lift of $X$ to a geometric $p$-rough path $\mathbf{X}$ for any $p>2 \rho$. Observe that the covariance of Brownian motion has finite $\rho$-variation with $\rho=1$. As a more general example, a direct computation shows that fractional Brownian motion has finite $\rho$-variation with $\rho=1 /(2 H)$.

The assumption of $|R|_{\rho \text {-var }}<\infty$ has other benefits, notably the following embedding theorem [8. Since it is crucial for our purposes we repeat the short proof; as we apply it componentwise we can assume $d=1$.

Proposition 2. Let $R$ be the covariance of a real-valued centered Gaussian process. If $R$ is of finite $\rho$-variation, then $\mathcal{H} \hookrightarrow C^{\rho-v a r}$. More, precisely, for all $h \in \mathcal{H}$,

$$
|h|_{\rho-\operatorname{var} ;[s, t]} \leq \sqrt{\langle h, h\rangle_{\mathcal{H}}} \sqrt{R_{\rho-\operatorname{var} ;[s, t]^{2}}} .
$$

Proof. Every element $h \in \mathcal{H}$ can be written as $h_{t}=\mathbb{E}\left(Z X_{t}\right)$ for the Gaussian r.v. $Z=\xi(h)$. We may assume that $\langle h, h\rangle_{\mathcal{H}}=\mathbb{E}\left(Z^{2}\right)=1$. Let $\left(t_{j}\right)$ be a subdivision of $[s, t]$ and write $|x|_{l^{r}}=\left(\sum_{i} x_{i}^{r}\right)^{1 / r}$ for $r \geq 1$. If $\rho^{\prime}$ denote the Hölder conjugate of $\rho$, we have

$$
\begin{aligned}
& \left(\sum_{j}\left|h_{t_{j}, t_{j+1}}\right|^{\rho}\right)^{1 / \rho}=\sup _{\beta,|\beta|_{l \rho^{\prime}} \leq 1} \sum_{j} \beta_{j} h_{t_{j}, t_{j+1}}=\sup _{\beta,|\beta|_{l \rho^{\prime}} \leq 1} \mathbb{E}\left(Z \sum_{j} \beta_{j} X_{t_{j}, t_{j+1}}\right) \\
& \leq \sup _{\beta,|\beta|_{l \rho^{\prime}} \leq 1} \sqrt{\sum_{j, k} \beta_{j} \beta_{k} \mathbb{E}\left(X_{t_{j}, t_{j+1}} X_{t_{k}, t_{k+1}}\right)} \\
& \leq \sup _{\beta,|\beta|_{l \rho^{\prime}} \leq 1} \sqrt{\left(\sum_{j, k}\left|\beta_{j}\right|^{\rho^{\prime}}\left|\beta_{k}\right|^{\rho^{\prime}}\right)^{\frac{1}{\rho^{\prime}}\left(\sum_{j, k}\left|\mathbb{E}\left(X_{t_{j}, t_{j+1}} X_{t_{k}, t_{k+1}}\right)\right|^{\rho}\right)^{\frac{1}{\rho}}}} \\
& \leq\left(\sum_{j, k}\left|\mathbb{E}\left(X_{t_{j}, t_{j+1}} X_{t_{k}, t_{k+1}}\right)\right|^{\rho}\right)^{1 /(2 \rho)} \leq \sqrt{R_{\rho-\operatorname{var} ;[s, t]^{2}}}
\end{aligned}
$$

Optimizing over all subdivision $\left(t_{j}\right)$ of $[s, t]$, we obtain our result. 
One observes that for Brownian motion $(\rho=1)$ this embedding is sharp. Furthermore, the rough path translation $T_{h} \mathbf{X}$, which involves Young integrals, makes sense if $\rho<\rho^{*}=3 / 2$. (This critical value comes from $1 / \rho+1 /(2 p) \sim 1 / \rho+1 /(2 \rho)=$ $3 /(2 \rho)$ and equating to 1.) The moral is that one can take deterministic directional derivatives in Cameron-Martin directions as long as $\rho<3 / 2$. (In passing, we see that the effective tangent space to Gaussian RDE solutions is strictly larger than the usual Cameron-Martin space as long as $\rho<3 / 2$. In the special case of Stratonovich SDEs a related result predates rough path theory and goes back to Kusuoka [15].) For this reason we will assume

$$
\rho<3 / 2
$$

for the remainder of this paper. This entails that we are dealing with geometric $p$-rough paths $\mathbf{X}$ for which we may assume

$$
p \in(2 \rho, 3) .
$$

Definition 1 ([13], 24, Section 4.1.3], [30, Section 3.3]). Given an abstract Wiener space $(W, \mathcal{H}, \mu)$, a random variable (i.e. measurable map) $F: W \rightarrow \mathbb{R}$ is continuously $\mathcal{H}$-differentiable, in symbols $F \in C_{\mathcal{H}}^{1}$, if for $\mu$-almost every $\omega$, the map

$$
h \in \mathcal{H} \mapsto F(\omega+h)
$$

is continuously Fréchet differentiable. A vector-valued r.v. $F=\left(F^{1}, \ldots, F^{e}\right): W \rightarrow$ $\mathbb{R}^{e}$ is continuously $\mathcal{H}$-differentiable iff each $F^{i}$ is continuously $\mathcal{H}$-differentiable. In particular, $\mu$-almost surely, $D F(\omega)=\left(D F^{1}(\omega), \ldots, D F^{e}(\omega)\right)$ is a linear bounded map from $\mathcal{H} \rightarrow \mathbb{R}^{e}$

Remark 1. (1) The notion of continuous $\mathcal{H}$-differentiability was introduced in [13] and plays a fundamental role in the study of the transformation of measure on Wiener space. Integrability properties of $F$ and $D F$ aside, $C_{\mathcal{H}}^{1}$-regularity is stronger than Malliavin differentiability in the usual sense. Indeed, by [24, Thm 4.1.3] (see also [13, [30, Section 3.3]) $C_{\mathcal{H}}^{1}$ implies $\mathbb{D}_{\text {loc }}^{1,2}$-regularity, where the definition of $\mathbb{D}_{\text {loc }}^{1,2}$ is based on the commonly used Shigekawa Sobolev space $\mathbb{D}^{1, p}$. (Our notation here follows [24, Sec. 1.2, 1.3.4]). This remark will be important to us since it justifies the use of Bouleau-Hirsch's criterion (e.g. [24, Section 2.1.2]) for establishing absolute continuity of $F$ (cf. the proof of Theorem 1).

(2) Although not relevant to the sequel of this paper, it is interesting to compare $C_{\mathcal{H}^{1}}^{1}$-regularity with the Kusuoka-Stroock Sobolev spaces. Following [14, 16] one defines $\tilde{\mathbb{D}}^{1, p}$ as the space of random-variables $F$ which are (i) ray-absolutely-continuous (RAC) in the sense that for every $h \in \mathcal{H}$ there is an absolutely continuous version of the process $\{F(\omega+t h): t \in \mathbb{R}\}$; (ii) stochastically Gateaux differentiable (SGD) in the sense that there exists an $\mathcal{H}$-valued r.v. $\tilde{D} F$ such that for every $h \in \mathcal{H}$,

$$
(F(\omega+t h)-F(\omega)) / t \rightarrow\langle\tilde{D} F, h\rangle_{\mathcal{H}} \text { as } t \rightarrow 0
$$

probability with respect to $\mu$; and (iii) such that $F \in L^{p}$ and $\tilde{D} F \in L^{p}(\mathcal{H})$.

From Sugita [27] it is known that $\tilde{\mathbb{D}}^{1, p}=\mathbb{D}^{1, p}$, at least for $p \in(1, \infty)$. Since $C_{\mathcal{H}^{-}}^{1}$ regularity is a local property, it has nothing to say about the integrability property (iii), but it does imply a fortiori the regularity properties (i) and (ii). Indeed, (i) is trivially satisfied (without the need of $h$-dependent modifications!). As for (ii), $\tilde{D} F$ is given by the Fréchet differential $D F(\omega)$ of $h \in \mathcal{H} \mapsto F(\omega+h)$, and the convergence (3.1) holds not only in probability but $\mu$-almost surely. 
Proposition 3. Let $\rho<3 / 2$. For fixed $t \geq 0$, the $\mathbb{R}^{e}$-valued random variable

$$
\omega \mapsto U_{t \leftarrow 0}^{\mathbf{X}(\omega)}\left(y_{0}\right)
$$

is continuously $\mathcal{H}$-differentiable.

Proof. Choose $p>2 \rho$ such that $1 / p+1 / \rho>1$. We may assume that $\mathbf{X}(\omega)$ has been defined so that $\mathbf{X}(\omega)$ is a geometric $p$-rough path for every $\omega \in W$. Let us also recall for $h \in \mathcal{H} \subset C^{\rho \text {-var }}$ that the translation $T_{h} \mathbf{X}(\omega)$ can be written (for $\omega$ fixed!) in terms of $\mathbf{X}(\omega)$ and cross-integrals between $\pi_{1}\left(\mathbf{X}_{0, .}\right)=: X \in C^{p \text {-var }}$ and $h$. (These integrals are well-defined Young-integrals.) Thanks to the definition of $\mathbf{X}(\omega)$ as the limit in probability of piecewise linear approximations to $X$ and its iterated integrals (cf. [8]) and basic continuity properties of Young integrals, we see that the event

$$
\left\{\omega: \mathbf{X}(\omega+h) \equiv T_{h} \mathbf{X}(\omega) \text { for all } h \in \mathcal{H}\right\}
$$

has probability one. We show that $h \in \mathcal{H} \mapsto U_{t \leftarrow 0}^{\mathbf{X}(\omega+h)}\left(y_{0}\right)$ is continuously Fréchet differentiable for every $\omega$ in the above set of full measure. By basic facts of Fréchet theory, we must show (a) Gateaux differentiability and (b) continuity of the Gateaux differential.

Ad (a): Using $\mathbf{X}(\omega+g+h) \equiv T_{g} T_{h} \mathbf{X}(\omega)$ for $g, h \in \mathcal{H}$ it suffices to show Gateaux differentiability of $U_{t \leftarrow 0}^{\mathbf{X}(\omega+\cdot)}\left(y_{0}\right)$ at $0 \in \mathcal{H}$. For fixed $t$, define

$$
Z_{i, s} \equiv J_{t \leftarrow s}^{\mathbf{X}}\left(V_{i}\left(U_{s \leftarrow 0}^{\mathbf{X}}\right)\right) \text {. }
$$

Note that $s \mapsto Z_{i, s}$ is of finite $p$-variation. We have, with implicit summation over $i$,

$$
\begin{aligned}
\left|D_{h} U_{t \leftarrow 0}^{\mathbf{X}}\left(y_{0}\right)\right| & =\left|\int_{0}^{t} J_{t \leftarrow s}^{\mathbf{X}}\left(V_{i}\left(U_{s \leftarrow 0}^{\mathbf{X}}\right)\right) d h_{s}^{i}\right| \\
& =\left|\int_{0}^{t} Z_{i} d h^{i}\right| \\
& \leq c\left(|Z|_{p-\mathrm{var}}+|Z(0)|\right) \times|h|_{\rho-\mathrm{var}} \\
& \leq c\left(|Z|_{p-\mathrm{var}}+|Z(0)|\right) \times|h|_{\mathcal{H}} .
\end{aligned}
$$

Hence, the linear map $D U_{t \leftarrow 0}^{\mathbf{X}}\left(y_{0}\right): h \mapsto D_{h} U_{t \leftarrow 0}^{\mathbf{X}}\left(y_{0}\right) \in \mathbb{R}^{e}$ is bounded, and each component is an element of $\mathcal{H}^{*}$. We just showed that

$$
h \mapsto\left\{\frac{d}{d \varepsilon} U_{t \leftarrow 0}^{T_{\varepsilon h} \mathbf{X}(\omega)}\left(y_{0}\right)\right\}_{\varepsilon=0}=\left\langle D U_{t \leftarrow 0}^{\mathbf{X}(\omega)}\left(y_{0}\right), h\right\rangle_{\mathcal{H}}
$$

and hence

$$
h \mapsto\left\{\frac{d}{d \varepsilon} U_{t \leftarrow 0}^{\mathbf{X}(\omega+\varepsilon h)}\left(y_{0}\right)\right\}_{\varepsilon=0}=\left\langle D U_{t \leftarrow 0}^{\mathbf{X}(\omega)}\left(y_{0}\right), h\right\rangle_{\mathcal{H}}
$$

emphasizing again that $\mathbf{X}(\omega+h) \equiv T_{h} \mathbf{X}(\omega)$ almost surely for all $h \in \mathcal{H}$ simultaneously. Repeating the argument with $T_{g} \mathbf{X}(\omega)=\mathbf{X}(\omega+g)$ shows that the Gateaux differential of $U_{t \leftarrow 0}^{\mathbf{X}(\omega+\cdot)}$ at $g \in \mathcal{H}$ is given by

$$
D U_{t \leftarrow 0}^{\mathbf{X}(\omega+g)}=D U_{t \leftarrow 0}^{T_{g} \mathbf{X}(\omega)} .
$$

(b) It remains to be seen that $g \in \mathcal{H} \mapsto D U_{t \leftarrow 0}^{T_{g} \mathbf{X}(\omega)} \in L\left(\mathcal{H}, \mathbb{R}^{e}\right)$, the space of linear bounded maps equipped with operator norm, is continuous. To this end, 
assume $g_{n} \rightarrow_{n \rightarrow \infty} g$ in $\mathcal{H}$ (and hence in $C^{\rho \text {-var }}$ ). Continuity properties of the Young integral imply continuity of the translation operator viewed as the map $h \in C^{\rho \text {-var }} \longmapsto T_{h} \mathbf{X}(\omega)$ (see [21]) and so

$$
T_{g_{n}} \mathbf{X}(\omega) \rightarrow T_{g} \mathbf{X}(\omega)
$$

in the $p$-variation rough path metric. The point here is that

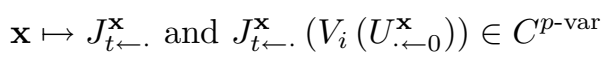

depends continuously on $\mathbf{x}$ with respect to the $p$-variation rough path metric: using

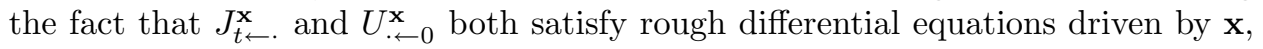
this is just a consequence of Lyons' limit theorem (the universal limit theorem of rough path theory). We apply this with $\mathbf{x}=\mathbf{X}(\omega)$, where $\omega$ remains a fixed element in (3.2). It follows that

$$
\left\|D U_{t \leftarrow 0}^{T_{g_{n}} \mathbf{X}(\omega)}-D U_{t \leftarrow 0}^{T_{g} \mathbf{X}(\omega)}\right\|_{o p}=\sup _{h:|h|_{\mathcal{H}}=1}\left|D_{h} U_{t \leftarrow 0}^{T_{g_{n}} \mathbf{X}(\omega)}-D_{h} U_{t \leftarrow 0}^{T_{g} \mathbf{X}(\omega)}\right|
$$

and defining $Z_{i}^{g}(s) \equiv J_{t \leftarrow s}^{T_{g} \mathbf{X}(\omega)}\left(V_{i}\left(U_{s \leftarrow 0}^{T_{g} \mathbf{X}(\omega)}\right)\right)$, and similarly $Z_{i}^{g_{n}}(s)$, the same reasoning as in part (a) leads to the estimate

$$
\left\|D U_{t \leftarrow 0}^{T_{g_{n}} \mathbf{X}(\omega)}-D U_{t \leftarrow 0}^{T_{g} \mathbf{X}(\omega)}\right\|_{o p} \leq c\left(\left|Z^{g_{n}}-Z^{g}\right|_{p-\text { var }}+\left|Z^{g_{n}}(0)-Z^{g}(0)\right|\right) .
$$

From the explanations just given this tends to zero as $n \rightarrow \infty$, which establishes continuity of the Gateaux differential, as required, and the proof is finished.

Definition $2\left([28,[24,22])\right.$. Given a continuously $\mathcal{H}$-differentiabl $\varrho^{3}$ r.v. $F=$ $\left(F^{1}, \ldots, F^{e}\right): W \rightarrow \mathbb{R}^{e}$ the Malliavin covariance matrix is the random matrix given by

$$
\sigma(\omega):=\left(\left\langle D F^{i}, D F^{j}\right\rangle_{\mathcal{H}}\right)_{i, j=1, \ldots, e} \in \mathbb{R}^{e \times e} .
$$

We call $F$ weakly non-degenerate if $\operatorname{det}(\sigma) \neq 0$ almost surely.

We now give an integral representation of the Malliavin covariance matrix of $Y_{t} \equiv$ $U_{t \leftarrow 0}^{\mathbf{X}(\omega)}\left(y_{0}\right)$, the solution to the RDE driven by $\mathbf{X}(\omega)$, and the lift of $\left(X^{1}, \ldots, X^{d}\right)$, along vector fields $\left(V_{1}, \ldots, V_{d}\right)$, in terms of 2D Young integrals [31, 29, 8].

Proposition 4. Let $\sigma_{t}=\left(\left\langle D Y_{t}^{i}, D Y_{t}^{j}\right\rangle_{\mathcal{H}}: i, j=1, \ldots, e\right)$ denote the Malliavin covariance matrix of $Y_{t} \equiv U_{t \leftarrow 0}^{\mathbf{X}(\omega)}\left(y_{0}\right)$, the $R D E$ solution of $d Y=V(Y) d \mathbf{X}(\omega)$. In the notation of Section 2.2 we have

$$
\left(\left\langle D Y_{t}^{i}, D Y_{t}^{j}\right\rangle_{\mathcal{H}}\right)_{i, j=1, \ldots, e}=\sum_{k=1}^{d} \int_{0}^{t} \int_{0}^{t} J_{t \leftarrow s}^{\mathbf{X}}\left(V_{k}\left(Y_{s}\right)\right) \otimes J_{t \leftarrow s^{\prime}}^{\mathbf{X}}\left(V_{k}\left(Y_{s^{\prime}}\right)\right) d R^{(k)}\left(s, s^{\prime}\right) .
$$

Proof. Let $\left(h_{n}^{(k)}: n\right)$ be an ONB of $\mathcal{H}^{(k)}$. It follows that $\left(h_{n}^{(k)}: n=1,2, \ldots ; k=1, \ldots, d\right)$ is an $\mathrm{ONB}$ of $\mathcal{H}=\bigoplus_{i=1}^{d} \mathcal{H}^{(k)}$ where we identify

$$
h_{n}^{(1)} \in \mathcal{H}^{(1)} \equiv\left(\begin{array}{c}
h_{n}^{(1)} \\
0 \\
\ldots \\
0
\end{array}\right) \in \mathcal{H}
$$

\footnotetext{
${ }^{3} D_{\text {loc }}^{1, p}$ is enough to guarantee the existence of an $\mathcal{H}$-valued derivative.
} 
and similarly for $k=2, \ldots, d$. From Parseval's identity,

$$
\begin{aligned}
\sigma_{t} & =\left(\left\langle D Y_{t}^{i}, D Y_{t}^{j}\right\rangle_{\mathcal{H}}\right)_{i, j=1, \ldots, e} \\
& =\sum_{n, k}\left\langle D Y_{t}, h_{n}^{(k)}\right\rangle_{\mathcal{H}} \otimes\left\langle D Y_{t}, h_{n}^{(k)}\right\rangle_{\mathcal{H}} \\
& =\sum_{k} \sum_{n} \int_{0}^{t} J_{t \leftarrow s}^{\mathbf{X}}\left(V_{k}\left(Y_{s}\right)\right) d h_{n, s}^{(k)} \otimes \int_{0}^{t} J_{t \leftarrow s}^{\mathbf{X}}\left(V_{k}\left(Y_{s}\right)\right) d h_{n, s}^{(k)} \\
& =\sum_{k} \int_{0}^{t} \int_{0}^{t} J_{t \leftarrow s}^{\mathbf{X}}\left(V_{k}\left(Y_{s}\right)\right) \otimes J_{t \leftarrow s^{\prime}}^{\mathbf{X}}\left(V_{k}\left(Y_{s^{\prime}}\right)\right) d R^{(k)}\left(s, s^{\prime}\right) .
\end{aligned}
$$

For the last step we used the fact that

$$
\sum_{n} \int_{0}^{T} f d h_{n} \int_{0}^{T} g d h_{n}=\int_{0}^{T} \int_{0}^{T} f(s) g(t) d R(s, t)
$$

whenever $f=f(t, \omega)$ and $g$ are such that the integrals are a.s. well-defined Youngintegrals. The proof is a consequence of $R(s, t)=\mathbb{E}\left(X_{s} X_{t}\right)$ and the $L^{2}$-expansion of the Gaussian process $X$,

$$
X(t)=\sum_{n} \xi\left(h_{n}\right) h_{n}(t)
$$

where $\xi\left(h_{n}\right)$ form an IID family of standard Gaussians.

Two special cases are worth considering: in the case of Brownian motion $d R\left(s, s^{\prime}\right)$ is a Dirac measure on the diagonal $\left\{s=s^{\prime}\right\}$ and the double integral reduces to a (well-known) single integral expression. In the case of fractional Brownian motion with $H>1 / 2$ it suffices to take the $\partial^{2} /(\partial s \partial t)$ derivative of $R_{H}(s, t)=$ $\left(t^{2 H}+s^{2 H}-|t-s|^{2 H}\right) / 2$ to see that

$$
d R_{H}\left(s, s^{\prime}\right) \sim|t-s|^{2 H-2} d s d t
$$

which is integrable iff $2 H-2>-1$ or $H>1 / 2$. (The resulting double-integral representation of the Malliavin covariance is also well-known and appears, for instance, in 25, 26, 1.)

\section{EXISTEnce OF A DENSity FOR Gaussian RDEs}

We remain in the framework of the previous sections, where $Y_{t}(\omega) \equiv U_{t \leftarrow 0}^{\mathbf{X}(\omega)}\left(y_{0}\right)$ denotes the (random) RDE solution driven a Gaussian rough path $\mathbf{X}$, the natural lift of a continuous, centered Gaussian process with independent components $X=$ $\left(X^{1}, \ldots, X^{d}\right)$ started at zero. Under the standing assumption of finite $\rho$-variation of the covariance, $\rho<3 / 2$, we know that $\mathbf{X}(\omega)$ is a.s. a geometric $p$-rough path for $p \in(2 \rho, 3)$. Recall that this means that $\mathbf{X}$ can be viewed as a path in $G^{2}\left(\mathbb{R}^{d}\right)$, the step-2 nilpotent group over $\mathbb{R}^{d}$, of finite $p$-variation relative to the CarnotCaratheodory metric on $G^{2}\left(\mathbb{R}^{d}\right)$.

Condition 1. Ellipticity assumption on the vector fields: The vector fields $V_{1}, \ldots, V_{d}$ span the tangent space at $y_{0}$. 
Condition 2. Non-degeneracy of the Gaussian process on $[0, T]$ : Fix $T>0$. Let $f=\left(f_{1}, \ldots, f_{d}\right):[0, T] \rightarrow \mathbb{R}^{d}$ be of finite $p$-variation, $1 / p+1 / \rho>1$, so that

$$
\int_{0}^{T} f d h \equiv \sum_{k=1}^{d} \int_{0}^{T} f_{k} d h^{k}
$$

exists in Riemann-Stieltjes sense for all $h \in \mathcal{H} \hookrightarrow C^{\rho \text {-var }}$. We say that nondegeneracy holds on $[0, T]$ if

$$
\left(\int_{0}^{T} f d h=0 \forall h \in \mathcal{H}\right) \Longrightarrow f \equiv 0 \text { a.e. }
$$

Note that non-degeneracy on $[0, T]$ implies non-degeneracy on $[0, t]$ for any $t \in$ $(0, T]$. If $h$ is absolutely continuous then one has $\int_{0}^{T} f d h=\int_{0}^{T} f_{t} \dot{h}_{t} d t$ where $\dot{h}$ denote the $L^{1}$-derivative of $h$. Typically, condition 2 is checked by exhibiting a subset $S \subset \mathcal{H}$ such that $\dot{S} \cap L^{2}$ is dense in $L^{2}=L^{2}\left([0, T], \mathbb{R}^{d}\right)$. Indeed, one then has that $f$ is orthogonal to a dense subset in $L^{2}$, hence zero almost everywhere on $[0, T]$. An illustrative example is given by the Brownian bridge, ruled out by our condition when returning to the origin at time $T$ or earlier. On the other hand, a Brownian bridge which returns to zero after time $T$ is non-degenerate on $[0, T]$. The following lemma contains a few ramifications concerning condition 2 . Since $\mathcal{H}=\oplus_{k=1}^{d} \mathcal{H}^{(k)}$ there is no loss in generality in assuming $d=1$.

Lemma 1. (i) Condition 2 applies in particular for $f \in C^{p \text {-var }}$ for $p>2 \rho$ small enough.

(ii) The requirement that $\int f d h=0 \forall h \in \mathcal{H}$ can be relaxed to the the quantifier "for all $h$ in some orthonormal basis of $\mathcal{H}$ ".

(iii) The non-degeneracy condition 2 implies that for all smooth $f \neq 0$, the zeromean Gaussian random variable $\int_{0}^{T} f d X$ (which exists as Young integral or via integration-by-parts) has positive definite variance.

(iv) The non-degeneracy condition 2 implies that for all times $0<t_{1}<\ldots<t_{n}<$ $T$ the covariance matrix of $\left(X_{t_{1}}, \ldots, X_{t_{n}}\right)$, that is, $\left(R\left(t_{i}, t_{j}\right)\right)_{i, j=1, \ldots, n}$, is (strictly) positive definite.

Proof. (i) Under our standing assumption $\rho<3 / 2$ we can pick $p>2 \rho$ such that $1 / p+1 / \rho>1$. Trivially, we can restrict attention to continuous functions $f$ of finite $p$-variation. (ii) Fix $f$ of finite $p$-variation and an orthonormal basis $\left(h_{k}\right) \subset \mathcal{H}$. We claim that

$$
\left\{\int_{0}^{T} f d h=0 \forall h \in \mathcal{H}\right\} \Leftrightarrow\left\{\int_{0}^{T} f d h_{k}=0 \forall k \in \mathbb{N}\right\}
$$

Only the "£" direction is non-trivial. Assuming $\int_{0}^{T} f d h_{k}=0$ for all $k$ implies $\int_{0}^{T} f d h^{[n]}=0$ for all $n$ where $h^{[n]} \equiv \sum_{k=1}^{n}\left\langle h_{k}, h\right\rangle h_{k}$ is the (truncated) Fourier expansion of $h$. It obviously converges in $\mathcal{H}$ (and hence also in $C^{\rho \text {-var }}$ ) to $h$ and we conclude by continuity of the Young integral. (iii) Let $f$ be smooth and assume that $Z:=\int_{0}^{T} f d X$ has variance zero. In other words, $Z(\omega)=0$ with probability one. By Cameron-Martin, $Z(\omega+h)=Z(\omega)+\int_{0}^{T} f d h$ is also zero with probability one and so $\int_{0}^{T} f d h=0$ for all $h \in \mathcal{H}$. By non-degeneracy, we see that $f$ must be identically equal to zero. In other words, for any smooth $f \neq 0$ the random variable $\int_{0}^{T} f d X$ has positive variance. (iv) Without loss of generality we assume 
that $X_{0}=0$; otherwise consider $\tilde{X}=X-X_{0}$ and observe that non-degeneracy of $X$ is equivalent to non-degeneracy of $\tilde{X}$. Let $\xi=\left(\xi^{1}, \ldots, \xi^{n}\right) \in \mathbb{R}^{n}$ be such that

$$
\sum_{i, j} \xi^{i} \xi^{j} R\left(t_{i}, t_{j}\right)=\mathbb{E}\left(\left|\sum_{i=1}^{n} \xi^{i} X_{t_{i}}\right|^{2}\right)=0
$$

Setting $t_{0}=0$ we have $\sum_{i=1}^{n} \xi^{i} X_{t_{i}}=\sum_{i=1}^{n} \zeta^{i}\left(X_{t_{i}}-X_{t_{i-1}}\right)$, where $\zeta$ is the linear image of $\xi$ under some (upper-triagonal) transformation, and by a Cameron-Martin argument, precisely as in (iii), $\sum_{i=1}^{n} \zeta^{i}\left(h_{t_{i}}-h_{t_{i-1}}\right)=0$ for all $h \in \mathcal{H}$. We can write this as the Riemann-Stieltjes integral of the step function $f=\sum \zeta^{i} 1_{\left(t_{i-1}, t_{i}\right]}$ against $d h$ and thus conclude, using our non-degeneracy condition, that $\zeta=0$. But this implies $\xi=0$ and the proof is finished.

Remark 2. The variance of $\int f d X$ can written as a 2D Young integral,

$$
\int_{[0, T]^{2}} f_{s} f_{t} d R(s, t) \text {. }
$$

To put the following result in context, recall that the covariance of Brownian motion, $(s, t) \mapsto \min (s, t)$, has finite 1-variation with $\rho=1$. For fractional Brownian motion with Hurst parameter $H$ one can take $\rho=1 / 2 H$. The following result then applies to fractional Brownian driving signals with $H>1 / 3$.

Theorem 1. Let $\mathbf{X}$ be a natural lift of a continuous, centered Gaussian process with independent components $X=\left(X^{1}, \ldots, X^{d}\right)$, with finite $\rho$-variation of the covariance, $\rho<3 / 2$ and non-degenerate in the sense of Condition 2. Let $V=\left(V_{1}, \ldots, V_{d}\right)$ be a collection of Lip ${ }^{3}$-vector fields on $\mathbb{R}^{e}$ which satisfy the ellipticity condition, Condition 1. Then the solution to the (random) $R D E$

$$
d Y=V(Y) d \mathbf{X}, Y(0)=y_{0} \in \mathbb{R}^{e}
$$

admits a density at all times $t \in(0, T]$ with respect to Lebesgue measure on $\mathbb{R}^{e}$.

Proof. Fix $t \in(0, T]$. From Proposition 3 we know that $U_{t \leftarrow 0}^{\mathbf{X}(\omega)} y_{0}=Y_{t}$ is continuously $\mathcal{H}$-differentiable. By a well-known criterion due to Bouleau-Hirsch 4 the proof is reduced to show a.s. invertibility of the Malliavin covariance matrix

$$
\sigma_{t}=\left(\left\langle D Y_{t}^{i}, D Y_{t}^{j}\right\rangle_{\mathcal{H}}\right)_{i, j=1, \ldots, e} \in \mathbb{R}^{e \times e} .
$$

Assume there exists a (random) vector $v \in \mathbb{R}^{e}$ which annihilates the quadratic form $\sigma_{t}$. Then 5

$$
0=v^{T} \sigma_{t} v=\left|\sum_{i=1}^{e} v_{i} D Y_{t}^{i}\right|_{\mathcal{H}}^{2}, \quad \text { and so } v^{T} D Y_{t} \equiv \sum_{i=1}^{e} v_{i} D Y_{t}^{i} \in 0 \in \mathcal{H} .
$$

By Propositions 1 and 3 ,

$$
\forall h \in \mathcal{H}: v^{T} D_{h} Y_{t}=\int_{0}^{t} \sum_{j=1}^{d} v^{T} J_{t \leftarrow s}^{\mathbf{X}}\left(V_{j}\left(Y_{s}\right)\right) d h_{s}^{j}=0,
$$

where the last integral makes sense as a Young integral since the (continuous) integrand has finite $p$-variation regularity. Noting that the non-degeneracy condition

\footnotetext{
${ }^{4}$ Combine the result of [Nualart, 24] 4.1.3] and [Nualart, 24] section 2].

${ }^{5}$ Upper $T$ denotes the transpose of a vector or matrix.
} 
on $[0, T]$ implies the same non-degeneracy condition on $[0, t]$, we see that the integrand in (4.1) must be zero on $[0, t]$ and the evaluation at time 0 shows that for all $j=1, \ldots, d$,

$$
v^{T} J_{t \leftarrow 0}^{\mathbf{X}}\left(V_{j}\left(y_{0}\right)\right)=0 .
$$

It follows that the vector $v^{T} J_{t \leftarrow 0}^{\mathbf{X}}$ is orthogonal to $V_{j}\left(y_{0}\right), j=1, \ldots, d$, and hence zero. Since $J_{t \leftarrow 0}^{\mathbf{X}}$ is invertible we see that $v=0$. The proof is finished.

The reader may be curious to hear about smoothness in this context. Adapting standard arguments would require $L^{p}(\Omega)$ estimates on the Jacobian of the flow $J_{t \leftarrow 0}^{\mathbf{X}(\omega)}$. Using the fact that it satisfies a linear RDE, $d J_{t \leftarrow t_{0}}^{\mathbf{X}}\left(y_{0}\right)=d \mathbf{M}^{\mathbf{X}} \cdot J_{t \leftarrow t_{0}}^{\mathbf{X}}\left(y_{0}\right)$, with $d \mathbf{M}^{\mathbf{X}}=V^{\prime}(Y) d \mathbf{X}$ one can see that

$$
\log \left|J_{t \leftarrow t_{0}}^{\mathbf{X}}\left(y_{0}\right)\right|=O\left(\|\mathbf{X}\|_{p \text {-var }}^{p}\right) .
$$

(This estimate appears in [26] for $p<2$ but can be seen [18, 9] to hold for all $p \geq 1$. We believe it to be optimal.) Using the Gauss tail of the homogenous $p$-variation norm of Gaussian rough paths (see [7, 8]) we see that $L^{q}$-estimates for all $q<\infty$ hold true when $p<2$, and this underlies to density results of [26, 1. On the other hand, for $p>2$ one cannot obtain $L^{q}$-estimates from (4.2), and further probabilistic input will be needed.

\section{ACKNOWLEDGEMENT}

We would like to thank James Norris for related discussions and an anonymous referee whose comments prompted a clarification in our discussion of $\mathcal{H}$-regularity. The second author is partially supported by a Leverhulme Research Fellowship.

\section{REFERENCES}

[1] Baudoin, F.; Hairer, M.: Hoermander's theorem for fractional Brownian motion, Probab. Theory Relat. Fields 139 (2007), no.3-4, 373-395. MR.2322701

[2] Bayraktar, E.; Horst, U.; Sircar R.: A Limit Theorem for Financial Markets with Inert Investors, Mathematics of Operations Vol 31, 2006 MR.2281230 (2007i:91062)

[3] Bell, D.: The Malliavin Calculus. Reprint of the 1987 edition, Dover Publications, Inc., Mineola, NY, 2006 MR2250060 (2007k:60003)

[4] Bismut, J.-M.: Large deviations and the Malliavin calculus. Progress in Mathematics, 45. Birkhäuser Boston, Inc., Boston, MA, 1984 MR755001 (86f:58150)

[5] Friz, P.; Lyons, T., Stroock, D.: Lévy's area under conditioning. Ann. Inst. H. Poincaré Probab. Statist. 42 (2006), no. 1, 89-101 MR2196973(2007i:60062)

[6] Friz, P.; Victoir, N.: Approximations of the Brownian rough path with applications to stochastic analysis. Ann. Inst. H. Poincaré Probab. Statist. 41 (2005), no. 4, 703-724 MR2144230 (2007e:60018)

[7] Friz, P.; Victoir, N.: A note on the notion of geometric rough paths. Probab. Theory Related Fields 136 (2006), no. 3, 395-416 MR2257130(2007k:60114)

[8] Friz, P.; Victoir, N.: Differential Equations Driven by Gaussian Signals I. arXiv-preprint.

[9] Friz, P.; Victoir, N.: Multidimensional Stochastic Processes as Rough Paths. Theory and Applications, Cambridge University Press (in preparation)

[10] Guasoni, P.: No Arbitrage with Transaction Costs with Fractional Brownian Motion and Beyond, Mathematical Finance 16 (2006) MR.2239592 (2007d:91091)

[11] Guasoni, P.; Rasonyi, M.; Schachermayer, W.: The Fundamental Theorem of Asset Pricing for Continuous Processes under Small Transaction Costs. Preprint (2007)

[12] Hairer, M.: Ergodicity of stochastic differential equations driven by fractional Brownian motion, Ann. Probab. 33 (2005), no 3, pp. 703-758 MR2123208 (2005k:60178) 
[13] Kusuoka, S.: The nonlinear transformation of Gaussian measure on Banach space and absolute continuity. I. J. Fac. Sci. Univ. Tokyo Sect. IA Math. 29 (1982), no. 3, 567-597 MR687592 (84j:60015a)

[14] Kusuoka, S.: Dirichlet forms and diffusion processes on Banach space. J. Fac. Sci. Univ. Tokyo Sect. IA Math. 29-1 (1982) MR657873 (83h:60082)

[15] Kusuoka, S.: On the regularity of solutions to SDEs. 'Asymptotic problem in probability theory: Wiener functionals and asymptotics', ed. K. D. Elworthy and N. Ikeda, Pitman Res. Notes Math. Ser., 284, pp.90-106, Longman Scientific \& Technica, 1993 MR1354163 (97a:60078)

[16] Kusuoka, S.; Stroock, D.: Applications of the Malliavin calculus I. Proceedings of the Taniguchi International Symposium on Stochastic Analysis, Katata and Kyoto, 1982. NorthHolland MR:780762 (86k:60100a)

[17] Kusuoka, S.; Stroock, D.: Applications of the Malliavin calculus III. J. Fac. Sci. Univ. Tokyo Sect. IA Math. 34 (1987), no. 2, 391-442 MR.914028 (89c:60093)

[18] Lyons, T.: Differential equations driven by rough signals, Rev. Mat. Iberoamericana 14 (1998), no. 2, 215-310 MR1654527(2000c:60089)

[19] Lyons, T.; Qian, Z.: Calculus of variation for multiplicative functionals, New trends in stochastic analysis (Charingworth, 1994), 348-374, World Sci. Publishing, River Edge, NJ, 1997 MR.1654380(2000k:60112)

[20] Lyons, T.; Qian, Z.: Flow of diffeomorphisms induced by a geometric multiplicative functional. Probab. Theory Related Fields 112 (1998), no. 1, 91-119 MR1646428 (99k:60153)

[21] Lyons, T.; Qian, Z.: System Control and Rough Paths, Oxford University Press, 2002. MR2036784 (2005f:93001)

[22] Malliavin, P.: Stochastic analysis. Grundlehren der Mathematischen Wissenschaften [Fundamental Principles of Mathematical Sciences], 313, Springer-Verlag, Berlin, 1997 MR 1450093 (99b:60073)

[23] McKean, H. P.: Stochastic Integrals, Academic Press, New York-London (1969). MR0247684 $(40: 947)$

[24] Nualart, D.: The Malliavin calculus and related topics. Second edition. Probability and its Applications (New York), Springer-Verlag, Berlin, 2006 MR2200233 (2006j:60004)

[25] Nualart, D.; Saussereau, B.: Malliavin calculus for stochastic differential equations driven by a fractional Brownian motion. Preprint (2005)

[26] Hu, Y.; Nualart, D.: Differential equations driven by Hölder continuous functions of order greater than 1/2; ArXiv (math.PR/0601628)

[27] Sugita, H.: On a characterization of the Sobolev spaces over an abstract Wiener Space. J. Fac. Sci. Univ. Tokyo Sect. IA Math. 25-1 (1985) 31-48 MR777244 (86j:60135)

[28] Shigekawa, I.: Stochastic analysis. Translated from the 1998 Japanese original by the author. Translations of Mathematical Monographs, 224. Iwanami Series in Modern Mathematics. American Mathematical Society, Providence, RI, 2004 MR2060917 (2005k:60002)

[29] Towghi, Nasser: Multidimensional extension of L. C. Young's inequality. JIPAM. J. Inequal. Pure Appl. Math., 3(2):Article 22, 13 pp. (electronic), 2002 MR1906391 (2003c:26035)

[30] Uestuenel, A. S.; Zakai, M.: Transformation of measure on Wiener space. Springer-Verlag, Berlin, 2000 MR 1736980 (2001g:60137)

[31] Young, L.C.: An inequality of Hölder type, connected with Stielties integration, Acta Math. 67, 251-282, 1936 MR1555421

Department of Pure Mathematics and Statistics, University of Cambridge, Wilberforce Road, Cambridge, CB3 0WB, United Kingdom

Current address: Mathematical Institute, University of Oxford, 24-29 St. Giles', Oxford, OX1 3LB, United Kingdom

Department of Pure Mathematics and Statistics, University of Cambridge, Wilberforce Road, Cambridge, CB3 0WB, United Kingdom 